\title{
Family History, Tobacco Smoking, and Risk of Ischemic Stroke
}

\author{
Mengyu Fan,, a,b Jun Lv, ${ }^{\mathrm{a}, \mathrm{c}, \mathrm{d}}$ Canqing Yu, ${ }^{\mathrm{a}}$ Yu Guo, ${ }^{\mathrm{e}}$ Zheng Bian, ${ }^{\mathrm{e}}$ Songchun Yang, ${ }^{\mathrm{a}}$ Ling Yang, \\ Yiping Chen, ${ }^{\mathrm{f}}$ Feifei Li, ${ }^{\mathrm{g}}$ Yaoming Zhai, ${ }^{\mathrm{g}}$ Ping Wang, ${ }^{\mathrm{h}}$ Junshi Chen, ${ }^{\mathrm{i}}$ Zhengming Chen, ${ }^{\mathrm{f}} \mathrm{Lu}$ Qi ${ }^{\mathrm{b}, \mathrm{j}}$ \\ Liming Li, ${ }^{a}$ on behalf of the China Kadoorie Biobank Collaborative Group* \\ aDepartment of Epidemiology and Biostatistics, School of Public Health, Peking University Health Science Center, Beijing, China \\ ${ }^{b}$ Department of Epidemiology, School of Public Health and Tropical Medicine, Tulane University, New Orleans, LA, USA \\ 'Key Laboratory of Molecular Cardiovascular Sciences (Peking University), Ministry of Education, Beijing, China

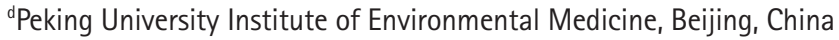 \\ ${ }^{e}$ Chinese Academy of Medical Sciences, Beijing, China \\ ${ }^{f}$ Clinical Trial Service Unit \& Epidemiological Studies Unit (CTSU), Nuffield Department of Population Health, University of Oxford, Oxford, UK \\ ${ }^{9} \mathrm{NCD}$ s Prevention and Control Department, Qingdao Centers for Disease Control, Qingdao, China \\ ${ }^{\mathrm{h} N C D s}$ Prevention and Control Department, Liuzhou Centers for Disease Control, Liuzhou, China \\ 'China National Center for Food Safety Risk Assessment, Beijing, China \\ jDepartment of Nutrition, Harvard School of Public Health, Boston, MA, USA
}

Background and Purpose Both genetic factors and smoking are associated with ischemic stroke (IS) risk. However, little is known about the potential interaction of these factors. We aimed to assess whether smoking and a positive family history interact to increase the risk of IS.

Methods The nationwide prospective study recruited 210,000 men and 300,000 women in 2004 to 2008 at ages 30 to 79 years. During 9.7 years of follow-up, we documented 16,923 and 20,656 incident IS cases in men and women without major chronic diseases at baseline, respectively. Multivariable Cox regression models were used to examine associations between family history and IS. Likelihood ratio tests were used to test the smoking-family history interactions on IS.

Results About $67.8 \%$ ( $n=135,168)$ of men ever smoked regularly compared with $2.7 \%(n=7,775)$ of women. Among men, a significant interaction between family history and smoking on IS was observed ( $P$ for interaction=0.03), with more pronounced association between family history and IS among ever-regular smokers (hazard ratio $[\mathrm{HR}], 1.21 ; 95 \%$ confidence interval $[\mathrm{CI}], 1.16$ to 1.27 ) than among never-smokers ( $\mathrm{HR}, 1.11 ; 95 \% \mathrm{Cl}, 1.01$ to 1.23$)$. The association between family history and IS among ex-smokers after more than 10 years of cessation ( $\mathrm{HR}, 1.01 ; 95 \% \mathrm{Cl}, 0.85$ to 1.20 ) appeared similar to that among never-smokers. Among women, a similar but not significant interaction between family history and smoking on IS was observed. Ever-regular smokers who had a family history of stroke had the highest risk of IS.

Conclusions Among Chinese men, the association of family history with IS was accentuated by smoking, and such accentuation tended to be lowered by cessation.

Keywords Family health; Stroke; Smoking; Cohort studies; Gene-environment interaction
Correspondence: Liming Li Department of Epidemiology and Biostatistics, School of Public Health, Peking University Health Science Center, 38 Xueyuan Road, Beijing 100191, China Tel: +86-10-82801528 (ext. 321)

Fax: +86-10-82801528 (ext. 321) E-mail:Imlee@vip.163.com

Co-correspondence: Lu Qi Department of Epidemiology, School of Public Health and Tropical Medicine, Tulane University, 1440 Canal Street, Suite 1724, New Orleans, LA 70112, USA

Tel: +1-504-988-7259

Fax: +1-504-988-7259

E-mail: lqi1@tulane.edu

Co-correspondence: Jun Lv Department of Epidemiology and Biostatistics, School of Public Health, Peking University Health Science Center, 38 Xueyuan Road, Beijing 100191, China Tel: +86-10-82801528 (ext. 322) Fax: +86-10-82801528 (ext. 322) E-mail: Ivjun@bjmu.edu.cn

Received: December 19, 2018

Revised: February 13, 2019

Accepted: April 18, 2019

*The members of steering committee and collaborative group are listed in the online-only Supplemental material. 


\section{Introduction}

Ischemic stroke (IS) is a complex disorder and the major cause of death and disability worldwide. ${ }^{12}$ In China, IS accounted for more than 0.7 million deaths in 2013, bringing an enormous public health burden. ${ }^{3}$ Notably, China is still experiencing increases in the incidence rate of IS. ${ }^{4}$ Smoking is a major modifiable risk factor for IS. ${ }^{5,6}$ Unfortunately, China is the world's largest tobacco consumer. According to the 2015 Chinese Adults Tobacco Survey Report, about 52.1\% of men and 2.7\% of women, accounting for more than 316 million people, were current smokers.' In China, even though the prevalence of smoking did not change in the past 5 years, the number of smokers increased as a consequence of expanding populations. ${ }^{89}$ In 2013, China was ranked highest for IS deaths attributable to tobacco consumption, which accounted for $20.3 \%$ of the population attributable risk for IS. ${ }^{1,10}$

Recently, compelling evidence has shown that genetic susceptibility may interact with lifestyle factors on chronic diseases risk. ${ }^{11,12}$ Previous studies found that the family history, a surrogate measure of genetic susceptibility, was associated with risk of stroke, ${ }_{1}^{13,14}$ and interacted with smoking on risk of cardiovascular outcomes such as myocardial infarction ${ }^{15}$ and subarachnoid hemorrhage ${ }^{16}$ However, little is known about the potential interactions between smoking and family history of stroke on the risk of IS, especially in Chinese.

Therefore, in the present study, we examined whether tobacco smoking modified the genetic susceptibility, estimated by a family history of stroke, in men and women from the China Kadoorie Biobank (CKB) study. We also prospectively examined the joint association of family history and tobacco smoking with IS risk.

\section{Methods}

\section{Study population}

Details of the CKB study have been described previously.17,18 Briefly, the CKB is a prospective cohort study of 512,715 adults aged 30 to 79 years recruited from 10 areas (five urban and five rural) of China. The baseline survey took place during 2004 to 2008. In each study assessment clinics, participants were interviewed by trained staff using laptop-based questionnaires, that covered demographic characteristics, socioeconomic status, lifestyle factors, general health, family history, mental health, and for women only, reproductive history. A range of physical measurements was undertaken by trained staff, including height, weight, and blood pressure. Blood samples were collected from each participant. All participants provided writ- ten informed consent. The Ethical Review Committee of the Chinese Center for Disease Control and Prevention (Beijing, China) and the Oxford Tropical Research Ethics Committee, University of Oxford (United Kingdom) approved the study.

For the present analysis, we excluded participants with previously diagnosed heart disease $(n=15,472)$, stroke $(n=8,884)$, or cancer $(n=2,577)$, and those who had missing data for body mass index $(n=2)$ or were lost to follow-up shortly after baseline $(n=1)$. The final analysis included 487,198 participants.

\section{Assessment of family history}

The baseline questionnaire included a series of three questions regarding family history of stroke, which asked participant if their biological father, mother, or siblings ever had "stroke." For siblings, the number with stroke was recorded. For each participant, the first-degree family members' stroke history had been collected. In this study, a participant was classified as "having a family history of stroke" if they reported at least one first degree relatives had stroke. First-degree relatives included fathers, mothers, and siblings.

\section{Assessment of smoking and other covariates}

Our baseline questionnaire collected smoking information including frequency, amount, and types of tobacco smoked (factory cigarettes, hand-rolled cigarettes, pipe or water pipe, cigars), depth of inhalation, and the ages at which participants started smoking regularly and stopped smoking, with additional information on the main reason for cessation (ill or stopped by choice) among those who had stopped smoking. Among current and former (or ex) regular smokers, the amount of tobacco smoked ( $\mathrm{g} /$ day) was calculated, assuming $1 \mathrm{~g}$ of tobacco per factory cigarette, and $2 \mathrm{~g}$ per cigar, with quantities smoked in pipes and hand-rolled cigarettes given as liang/month (1 liang equivalent to $50 \mathrm{~g}$ ). Smoking duration in years was derived from the age at starting smoking regularly to age at baseline (current smokers) or duration since quitting at baseline (ex-smokers).

For the present study, participants were categorized into four groups according to the smoking status in line with previous CKB studies. ${ }^{19}$ Never-smokers were defined as those who had not smoking at recruitment and who had not smoked more than 100 cigarettes during their lifetime. Ever-regular smokers were defined as those who had ever smoked at least one cigarette or equivalent per day for at least 6 months, or who had stopped smoking at least 6 months before recruitment because of ill. Regular smokers who had stopped smoking by choice for at least 6 months at baseline were classified as ex-smokers. Participants who did not meet the criteria for never-smoker, ever-regular smoker, or ex-smoker were classified as occasional smokers. 
The baseline questionnaire also included other covariates, such as sociodemographic characteristics (age, sex, education, marital status, and household income), lifestyle behaviors (physical activity, alcohol consumption, intakes of red meat, fresh fruits, and vegetables), and menopausal status (for women). Trained staff measured weight and height using calibrated instruments. Prevalent diabetes was defined as measured fasting blood glucose $\geq 7.0 \mathrm{mmol} / \mathrm{L}$, measured random blood glucose $\geq 11.1 \mathrm{mmol} / \mathrm{L}$, or self-reported diagnosis of diabetes. Prevalent hypertension was defined as measured systolic blood pressure $\geq 140 \mathrm{~mm} \mathrm{Hg}$, measured diastolic blood pressure $\geq 90$ $\mathrm{mm} \mathrm{Hg}$, self-reported diagnosis of hypertension, or self-reported use of antihypertensive agents at baseline.

\section{Follow-up for incident IS}

All the participants were followed up from the time they enrolled in the study. We ascertained the vital status of each participant through the linkage with established chronic disease registries, and the disease surveillance points system in the study areas, as well as the national health insurance system. Almost all the participants (>98\%) across the 10 study areas had been successfully linked to the health insurance databases. Annual active followup was performed by checking against local residential and health insurance records and was confirmed by street committees or village administrators. Fatal and non-fatal events were coded by the trained staff, blinded to baseline information, using the International Classification of Diseases, 10th Revision. All reported fatal or non-fatal major diseases events (including stroke) from different sources were reviewed and integrated centrally by trained clinical staff, who were blinded to baseline information. By December 2018, we have retrieved medical records for 31,069 newly reported IS cases during follow-up, among which 21,426 (69\%) were confirmed as IS cases.

\section{Statistical analysis}

As the prevalence of smoking and the number of cigarettes smoked per day are much higher in men compared with women, all analyses were done separately in men and women. Percentages and mean \pm standard deviation of baseline characteristics were calculated across family history of stroke standardized by age and study area, when appropriate. We calculated person-years at risk from the baseline date to diagnosis of IS, death, loss to follow-up, or the censoring date (December 31, 2016), whichever occurred first. Cox regression was used to estimate the hazard ratios (HRs) of family history, with age as the underlying time scale and stratified jointly by 10 study areas and age at baseline in 5-year intervals. Multivariable models were adjusted for education (no formal school, primary school, middle school, high school, or college or university or higher), marital status (married, widowed, divorced/separated, or never married), household income $(<10,000,10,000$ to 19,999 , $\geq 20,000$ Chinese renminbi/yr), alcohol consumption (less than weekly, ex-regular drinkers, weekly; or $<15,15$ to 29,30 to 59 , or $\geq 60 \mathrm{~g} /$ day of pure alcohol), smoking status (never-smokers or occasional smokers, or ex-smokers, or current smokers of 1 to 14,15 to 24 , or $\geq 25$ cigarettes or equivalents per day), physical activity as metabolic equivalent of task hours per day, intake of red meat, fresh fruits, and vegetables (daily, 4 to 6 days/week, 1 to 3 days/week, monthly, or rarely or never), menopause status (for women only, premenopausal, perimenopause, postmenopausal, or missing), body mass index (in $\left.\mathrm{kg} / \mathrm{m}^{2}\right)$, and prevalent diabetes and hypertension at baseline (presence or absence).

Interactions between the family history of stroke and tobacco smoking on IS were tested by using likelihood ratio tests to compare models with and without cross-product terms (e.g., interaction term: family history of stroke $x$ smoking categories). The HRs for IS were also calculated by strata of smoking characteristic subgroups, using individuals who reported no family history of stroke as the reference group. We further examined the risk of incident IS according to the joint classification of family history and smoking, in which smoking variables were included two categories (never-smokers and ever-regular smokers). We performed all statistical analyses using Stata version 14.2 (StataCorp., College Station, TX, USA).

\section{Results}

\section{Population characteristics according to family history of stroke}

Overall, the mean baseline age was $51.5 \pm 10.5$ years, $67.8 \%$ $(n=135,168)$ of men ever smoked regularly compared with $2.7 \%(n=7,775)$ of women. Of the 199,240 men and 287,958 women included, $17.8 \%$ of men and $17.5 \%$ of women had a family history of stroke. Both men and women who had a family history of stroke were, on average, more likely to reside in the urban area, have a higher level of education, and have hypertension at baseline (Table 1).

\section{Family history of stroke and IS}

During 10 years of follow-up (4.7 million person years), we documented 16,923 incident IS cases in men and 20,656 in women. Multivariate-adjusted analyses showed that family history of stroke was associated with an increased IS risk (Supplementary Table 1). In the whole cohort, compared with participants who reported no family history of stroke, the adjusted HR for IS was 1.19 (95\% confidence interval [CI], 1.16 to 1.22) 
Table 1. Baseline characteristics of 487,198 participants according to family history of stroke

\begin{tabular}{|c|c|c|c|c|}
\hline \multirow{2}{*}{ Characteristic } & \multicolumn{2}{|c|}{ Family history in men $(n=199,240)$} & \multicolumn{2}{|c|}{ Family history in women $(n=287,958)$} \\
\hline & No & Yes & No & Yes \\
\hline No. of participants & $163,791(82.2)$ & $35,449(17.8)$ & $237,436(82.5)$ & $50,522(17.5)$ \\
\hline Age (yr) & $52.4 \pm 11.0$ & $52.1 \pm 9.8$ & $50.9 \pm 10.5$ & $51.3 \pm 9.4$ \\
\hline Urban area (\%) & 41.9 & 45.9 & 42.4 & 48.5 \\
\hline Middle school and above (\%) & 57.1 & 60.9 & 42.5 & 46.8 \\
\hline Married (\%) & 92.8 & 93.7 & 89.3 & 89.9 \\
\hline Household income $\geq 20,000$ yuan/y (\%) & 45.5 & 47.0 & 40.3 & 42.5 \\
\hline Postmenopausal (\%) & - & - & 50.8 & 50.6 \\
\hline \multicolumn{5}{|l|}{ Smoking category (\%) } \\
\hline Never-smokers & 14.4 & 13.3 & 95.1 & 94.9 \\
\hline Ex-smokers & 6.6 & 6.8 & 0.4 & 0.4 \\
\hline Occasional smokers & 11.4 & 11.2 & 1.8 & 2.0 \\
\hline Ever-regular smokers & 67.7 & 68.7 & 2.7 & 2.8 \\
\hline Weekly alcohol drinker (\%) & 33.7 & 35.1 & 2.1 & 2.1 \\
\hline \multicolumn{5}{|l|}{ Average weekly consumption (day)* } \\
\hline Red meat & 4.0 & 4.0 & 3.5 & 3.6 \\
\hline Fresh vegetable & 6.8 & 6.8 & 6.8 & 6.8 \\
\hline Fresh fruit & 2.2 & 2.3 & 2.7 & 2.9 \\
\hline Total physical activity (MET hr/day) & 22.5 & 23.0 & 20.8 & 20.9 \\
\hline Body mass index $\left(\mathrm{kg} / \mathrm{m}^{2}\right)$ & 23.3 & 23.6 & 23.7 & 24.0 \\
\hline Prevalent diabetes (\%) & 5.0 & 5.5 & 5.4 & 6.3 \\
\hline Prevalent hypertension (\%) & 34.5 & 42.2 & 30.9 & 38.3 \\
\hline
\end{tabular}

Values are presented as number (\%) or mean \pm standard deviation.

MET, metabolic equivalent.

*Average weekly consumptions of red meat, fresh vegetables, and fruits were calculated by assigning participants to the midpoint of their consumption category.

for those who reported a family history of stroke. The respective HRs were 1.19 (95\% $\mathrm{Cl}, 1.15$ to 1.23$)$ among men and 1.19 $(95 \% \mathrm{Cl}, 1.15$ to 1.23$)$ among women. There was no heterogeneity between men and women ( $P$ for interaction=0.92).

\section{Interactions between family history of stroke and smoking on IS risk}

Among men, we observed a significant interaction between family history of stroke and IS risk across the stratum for tobacco smoking ( $P$ for interaction=0.03), with a stronger association among ever-regular smokers than among never-smokers (Table 2). Compared with men who reported no family history of stroke, the adjusted HRs for those reported a family history of stroke were 1.11 (95\% Cl, 1.01 to 1.23) among never-smokers and $1.21(95 \% \mathrm{Cl}, 1.16$ to 1.27$)$ among ever-regular smokers. Among women, differences in the associations between family history and IS risk across the smoking status were observed, but the interaction was not significant ( $P$ for interaction $=0.49$ ). The respective HRs for those who reported a family history of stroke were $1.19(95 \% \mathrm{Cl}, 1.15$ to 1.23$)$ among never-smokers, and
$1.32(95 \% \mathrm{Cl}, 1.14$ to 1.53$)$ among ever-regular smokers. Likewise, compared with never-smokers, the adjusted HRs for everregular smokers were greater in those who reported a family history of stroke than those who did not (Table 3).

We further examined the interaction between each type of family history and the smoking history (Supplementary Table 2). We only found a significant interaction between parental history and smoking status among men. Nevertheless, the associations between each type of family history and IS risk seemed to be less evident in never-smokers compared with ever-regular smokers.

\section{Stratified analyses among smokers}

Among ever-regular smokers, we further performed stratified analyses according to baseline smoking characteristics: types of tobacco smoked, age started smoking, smoking duration, smoking amount, and depth of inhalation. Among men, the HRs for family history on IS were markedly higher among all subgroups compared with never-smokers (Figure 1), but did not significantly differ across different smoking characteristics 
Table 2. Risk of incident ischemic stroke according to family history of stroke, stratified by smoking category

\begin{tabular}{|c|c|c|c|c|c|c|c|}
\hline \multirow{3}{*}{ Variable } & \multicolumn{6}{|c|}{ Family history of stroke } & \multirow{3}{*}{$P_{\text {interaction }}$} \\
\hline & \multicolumn{3}{|c|}{ No } & \multicolumn{3}{|c|}{ Yes } & \\
\hline & Total no. & Cases & $\mathrm{HR}(95 \% \mathrm{Cl})$ & Total no. & Cases & HR $(95 \% \mathrm{Cl})$ & \\
\hline Men & & & & & & & 0.03 \\
\hline Never-smokers & 23,611 & 2,222 & Ref & 4,666 & 578 & $1.11(1.01-1.23)$ & \\
\hline Ex-smokers & 10,650 & 1,114 & Ref & 2,491 & 332 & $1.13(0.99-1.28)$ & \\
\hline Occasional smokers & 18,413 & 1,286 & Ref & 4,207 & 420 & $1.18(1.05-1.32)$ & \\
\hline Ever-regular smokers & 111,117 & 8,311 & Ref & 24,085 & 2,660 & $1.21(1.16-1.27)$ & \\
\hline Women & & & & & & & 0.49 \\
\hline Never-smokers & 225,929 & 14,453 & Ref & 47,891 & 4,615 & $1.19(1.15-1.23)$ & \\
\hline Ex-smokers & 848 & 133 & Ref & 196 & 37 & $1.04(0.70-1.54)$ & \\
\hline Occasional smokers & 4,228 & 304 & Ref & 1,049 & 125 & $1.20(0.95-1.50)$ & \\
\hline Ever-regular smokers & 6,431 & 733 & Ref & 1,386 & 256 & $1.32(1.14-1.53)$ & \\
\hline
\end{tabular}

Stratified by age and study area and adjusted for level of education; marital status; household income; alcohol consumption; physical activity; intake frequencies of red meat, fresh fruits, and vegetables; menopause status (only for women); body mass index; prevalent hypertension; prevalent diabetes.

$\mathrm{HR}$, hazard ratio; $\mathrm{Cl}$, confidence interval.

Table 3. Risk of incident ischemic stroke according to smoking category, stratified by family history of stroke

\begin{tabular}{llc}
\hline \multirow{2}{*}{ Variable } & \multicolumn{2}{c}{ Family history of CVD } \\
\cline { 2 - 3 } Men & No & Yes \\
Never-smokers & 1.00 & 1.00 \\
Ex-smokers & $0.99(0.92-1.07)$ & $1.05(0.92-1.19)$ \\
Occasional smokers & $0.99(0.92-1.06)$ & $1.03(0.91-1.17)$ \\
Ever-regular smokers & $1.17(1.11-1.23)$ & $1.30(1.19-1.43)$ \\
Women & & 1.00 \\
Never-smokers & 1.00 & $0.90(0.65-1.23)$ \\
Ex-smokers & $0.98(0.82-1.17)$ & $1.07(0.90-1.28)$ \\
Occasional smokers & $0.98(0.87-1.11)$ & $1.29(1.14-1.47)$ \\
Ever-regular smokers & $1.09(1.00-1.18)$ & \\
\hline
\end{tabular}

Values are presented as hazard ratio (95\% confidence interval). Stratified by age and study area and adjusted for level of education; marital status; household income; alcohol consumption; physical activity; intake frequencies of red meat, fresh fruits, and vegetables; menopause status (only for women); body mass index; prevalent hypertension; prevalent diabetes.

subgroups (all $P$ for interaction $>0.05$ ). Similar but more evident associations were observed among women.

Among ex-smokers, no statistically significant heterogeneity was observed in the associations between family history of stroke and IS by years of smoking cessation (Table 4). Nevertheless, the associations between family history of stroke and IS seemed to be less evident in ex-smokers with longer years since quitting. Among men, the HRs were $1.32(95 \% \mathrm{Cl}, 1.09$ to 1.59 ) among those who had stopped for less than 10 years, and $1.01(95 \% \mathrm{Cl}, 0.85$ to 1.20$)$ among those who had stopped for 10 years or more before baseline ( $P$ for interaction $=0.05$ ). Similar patterns were observed among women, with HRs of $1.01(95 \% \mathrm{Cl}, 0.55$ to 1.84$)$ and $0.92(95 \% \mathrm{Cl}, 0.51$ to 1.65$)$, re- spectively ( $P$ for interaction $=0.86)$.

\section{Joint association of family history of stroke and smoking with IS risk}

We further examined the joint association of family history and smoking with IS risk (Figure 2). The strongest association was observed in the combination of family history with ever-regular smoking. Compared with never-smokers who reported no family history of stroke, ever-regular smokers who reported a family history of stroke showed a $42 \%$ risk increasing in IS (HR, 1.42; $95 \% \mathrm{Cl}_{1} 1.34$ to 1.51 ) among men and a $43 \%$ risk increasing ( $\mathrm{HR}, 1.43 ; 95 \% \mathrm{Cl}, 1.26$ to 1.63$)$ among women, respectively. 


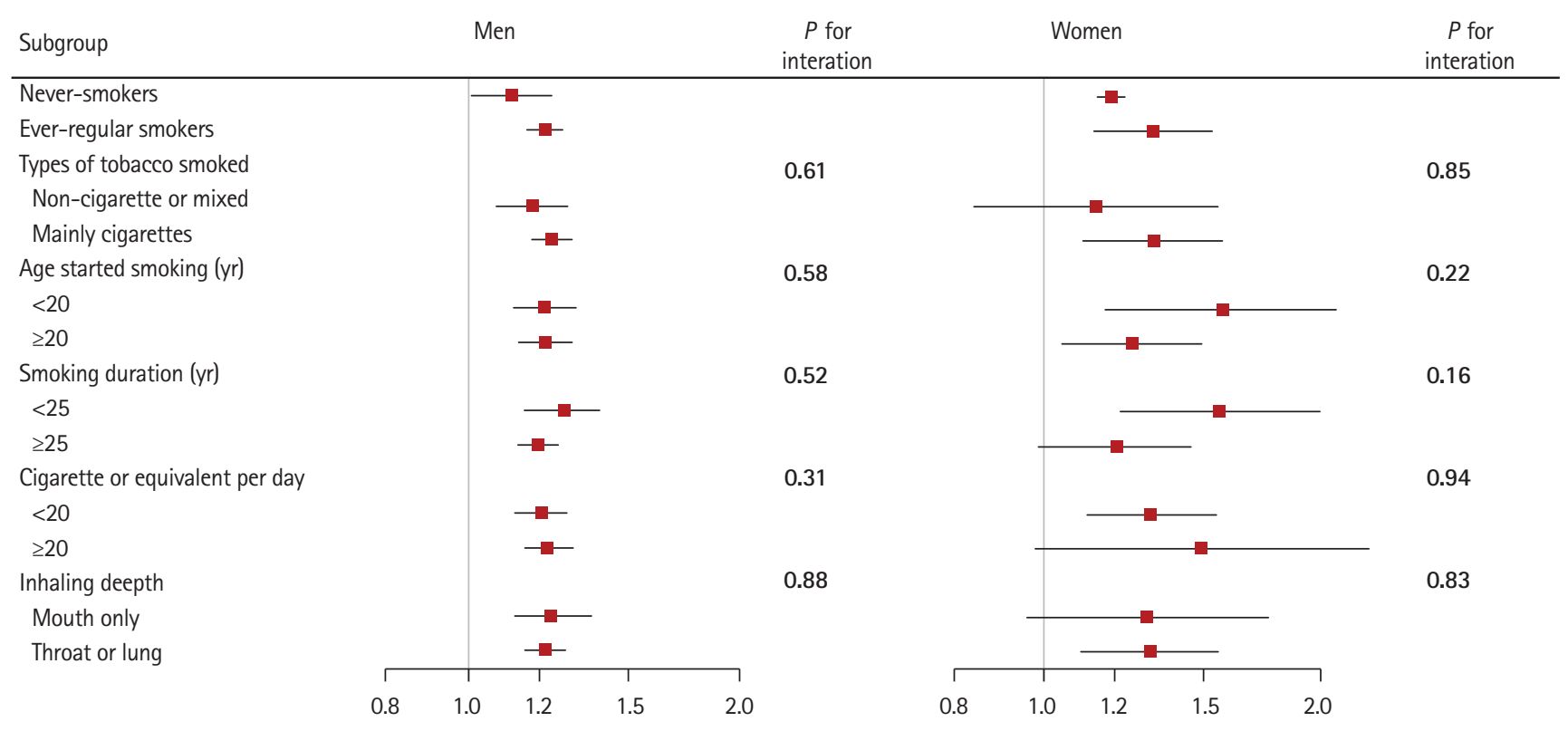

Figure 1. Subgroup analysis of associations between family history and ischemic stroke according to baseline smoking characteristics. Hazard ratios for ischemic stroke are for comparison of men and women who had a family history of stroke with those who had no family history of stroke. Ever-regular smokers excludes occasional smokers $(n=22,620)$ and ex-smokers who stopped by choice $(n=13,141)$. Tests for heterogeneity include only ever-regular smokers. Horizontal lines represent $95 \%$ confidence intervals.

Table 4. Risk of incident ischemic stroke according to family history of stroke, stratified by smoking stopped years

\begin{tabular}{|c|c|c|c|c|c|c|c|}
\hline \multirow{3}{*}{ Variable } & \multicolumn{6}{|c|}{ Family history of stroke } & \multirow{3}{*}{$P_{\text {interactio }}$} \\
\hline & \multicolumn{3}{|c|}{ No } & \multicolumn{3}{|c|}{ Yes } & \\
\hline & Total no. & Cases & $\mathrm{HR}(95 \% \mathrm{Cl})$ & Total no. & Cases & HR $(95 \% \mathrm{Cl})$ & \\
\hline Men & & & & & & & 0.05 \\
\hline Never-smokers & 23,611 & 2,222 & Ref & 4,666 & 578 & $1.11(1.01-1.23)$ & \\
\hline \multicolumn{8}{|l|}{ Ex-smokers } \\
\hline Stopped for $<10 \mathrm{yr}$ & 5,692 & 471 & Ref & 1,268 & 157 & $1.32(1.09-1.59)$ & \\
\hline Stopped for $\geq 10 \mathrm{yr}$ & 4,958 & 643 & Ref & 1,223 & 175 & $1.01(0.85-1.20)$ & \\
\hline Women & & & & & & & 0.86 \\
\hline Never-smokers & 225,929 & 14,453 & Ref & 47,891 & 4,615 & $1.19(1.15-1.23)$ & \\
\hline \multicolumn{8}{|l|}{ Ex-smokers } \\
\hline Stopped for $<10 \mathrm{yr}$ & 394 & 63 & Ref & 95 & 17 & $1.01(0.55-1.84)$ & \\
\hline Stopped for $\geq 10 \mathrm{yr}$ & 454 & 70 & Ref & 101 & 20 & $0.92(0.51-1.65)$ & \\
\hline
\end{tabular}

Adjusted for age; study area; level of education; marital status; household income; alcohol consumption; physical activity; intake frequencies of red meat, fresh fruits, and vegetables; menopause status (only for women); body mass index; prevalent hypertension; prevalent diabetes.

$\mathrm{HR}$, hazard ratio; $\mathrm{Cl}$, confidence interval.

\section{Discussion}

In this large prospective cohort of Chinese adults, we observed a significant interaction between smoking and family history of stroke on IS incidence risk among men. The association between family history of stroke and IS was more pronounced among ever-regular smokers than among never-smokers; whereas the association between family history and IS risk among ex-smokers after more than 10 years of cessation ap- peared similar to that among never-smokers. The interactions between smoking and family history of stroke were consistent across the smoking characteristic subgroups including types of tobacco smoked, age at starting, smoking duration, smoking amount, or depth of inhalation. Participants with a family history of stroke and reported ever-regular smoking had the greatest risk of IS.

Our study is the first to examine the interaction between smoking and family history of stroke on incident IS in a pro- 


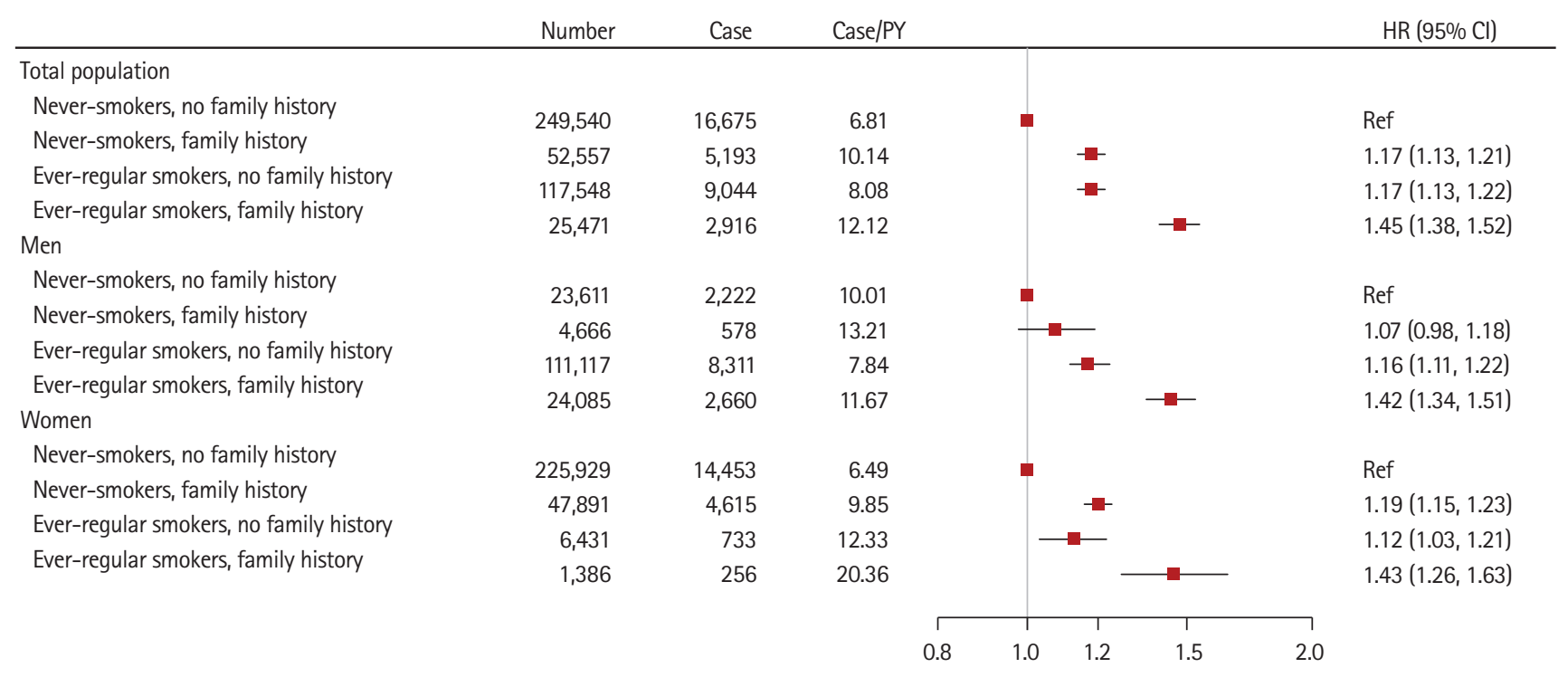

Figure 2. Risk of incident ischemic stroke according to family history of stroke and smoking status. Family history refers to family history of stroke. Model was stratified by age and study area and adjusted for level of education; marital status; household income; alcohol consumption; physical activity; intake frequencies of red meat, fresh fruits, and vegetables; menopause status (only for women); body mass index; prevalent hypertension; prevalent diabetes. Horizontal lines represent 95\% confidence intervals (Cls). HR, hazard ratio; PY, person-year.

spective cohort. Our findings are in line with several previous cross-sectional studies that reported the interactions between smoking and single genetic variants on IS..$^{20-22}$ Similar smokinggene interaction in relation to IS was also found in Chinese populations..$^{23-25}$ In our study, we found that the interaction between smoking and family history of stroke was particularly significant among men, even though a similar but not significant interaction pattern was also observed among women. The gender difference may partly due to the extremely small number of ever-regular smokers among women.

For the first time, we further provided data about the interplay between family history and different smoking characteristics on IS among ever-regular smokers. The associations between family history and IS were not significantly differ across different smoking characteristics subgroups. Our findings were weakened by the fact that the effect of family history on IS risk did not increase with greater amount smoked and increased smoking duration. Although numbers are small in the stratified analyses among smokers, our findings may imply that the smokers themselves differ from the never-smokers, and that in general, the effects of family history are minimal in neversmokers.

Our observations, together with previous reports, suggest that smoking may strengthen the deleterious effects of genetic variants on the development of IS. The potential mechanisms underlying the smoking-gene interactions remain unclear. Smoking is associated with endothelial cell dysfunction, ${ }^{26-29}$ in- creased activation of inflammation and coagulation, ${ }^{30}$ increased fibrinogen concentration and platelet aggregability, ${ }_{1}{ }^{31}$ as well as reduced fibrinolytic capacity, ${ }_{1}^{29}$ which may cause a procoagulant state and subsequently promote atherosclerosis and increase IS risk. Of note, all these alternations may overlap with pathways linking the genetic variations to the development of IS, making the interaction between smoking and genetic factors biologically feasible.

One of the novel findings of the present study is that the associations between family history and IS risk among ex-smokers after more than 10 years of cessation were similar to the level of never-smokers, indicating the risk conferred by an interaction may be lowered by quitting smoking. The present results strongly suggest that although the best public health advice remains not to start smoking at all, encouraging smokers to stop is crucial to reduce smoking-attributed IS risk, particularly among those with a family history of stroke. On the basis of the high smoking prevalence among Chinese, widespread smoking cessation is one of the most effective and cost-effective strategies to reduce IS risk over the next few decades. ${ }^{32}$

The major strengths of the present study included prospective design, large sample size, a population geographically spread across urban and rural China, and careful adjustment for nongenetic familial factors. Several limitations need to be acknowledged. The participants consisted of Chinese middleaged and older men and women. Thus the results may not be generalizable to other age or racial/ethnic groups. Moreover, 
smoking status and family history was self-reported, which might lead to some degree of misclassification. However, measurement errors in the prospective study design are more likely to be non-differential and therefore, would have biased the results toward the null. In addition, the family history of stroke is a relatively crude marker for genetic susceptibility. Future studies need to examine the gene-smoking interaction by using well-established IS-associated genetic variants. Finally, although we have adjusted various confounding variables, residual confounding is still possible.

\section{Conclusions}

In summary, we found the impact of family history on IS was accentuated by tobacco smoking; and such accentuation tended to be reversible after more than 10 years of cessation. Our findings reemphasize the importance of counseling of family members regarding smoking behavior, and considering geneenvironment interaction in the prevention of IS.

\section{Supplementary materials}

Supplementary materials related to this article can be found online at https://doi.org/10.5853/jos.2018.03566.

\section{Disclosure}

The authors have no financial conflicts of interest.

\section{Acknowledgments}

The most important acknowledgement is to the participants in the study and the members of the survey teams in each of the 10 regional centres, as well as to the project development and management teams based at Beijing, Oxford, and the 10 regional centres.

This work was supported by grants (2016YFC0900500, 2016YFC0900501, 2016YFC0900504) from the National Key R\&D Program of China. The CKB baseline survey and the first resurvey were supported by a grant from the Kadoorie Charitable Foundation in Hong Kong. The long-term follow-up is supported by grants from the UK Wellcome Trust (202922/Z/16/Z, 088158/ Z/09/Z, 104085/Z/14/Z), National Natural Science Foundation of China (81390540, 81390544, 81390541), and Chinese Ministry of Science and Technology (2011BAI09B01). Lu Qi is supported by grants from the National Heart, Lung, and Blood Institute (HL071981, HL034594, HL126024), the National Institute of Diabetes and Digestive and Kidney Diseases (DK115679, DK091718,
DK100383, DK078616). Mengyu Fan is a recipient of a scholarship under the China Scholarship Council (201706010313).

\section{References}

1. Bennett DA, Krishnamurthi RV, Barker-Collo S, Forouzanfar $\mathrm{MH}$, Naghavi $\mathrm{M}$, Connor $\mathrm{M}$, et al. The global burden of ischemic stroke: findings of the GBD 2010 study. Glob Heart 2014;9:107-112.

2. GBD 2015 Disease and Injury Incidence and Prevalence Collaborators. Global, regional, and national incidence, prevalence, and years lived with disability for 310 diseases and injuries, 1990-2015: a systematic analysis for the Global Burden of Disease Study 2015. Lancet 2016;388:1545-1602.

3. Zhou M, Wang H, Zhu J, Chen W, Wang L, Liu S, et al. Causespecific mortality for 240 causes in China during 1990-2013: a systematic subnational analysis for the Global Burden of Disease Study 2013. Lancet 2016;387:251-272.

4. Wang $W_{1}$, Jiang $B$, Sun $H, R u X$, Sun $D$, Wang $L$, et al. Prevalence, incidence, and mortality of stroke in China: results from a nationwide population-based survey of 480687 adults. Circulation 2017;135:759-771.

5. Wang J, Wen $X$, Li W, Li X, Wang Y, Lu W. Risk factors for stroke in the Chinese population: a systematic review and meta-analysis. J Stroke Cerebrovasc Dis 2017;26:509-517.

6. Boehme AK, Esenwa C, Elkind MS. Stroke risk factors, genetics, and prevention. Circ Res 2017;120:472-495.

7. Chinese Center for Disease Control and Prevention. 2015 Chinese adults tobacco survey report. http://www.notc.org. cn/gzdt/201512/t20151228_123959.html. 2015. Accessed April 23, 2019.

8. Ng M, Freeman MK, Fleming TD, Robinson M, Dwyer-Lindgren $L$, Thomson $\mathrm{B}$, et al. Smoking prevalence and cigarette consumption in 187 countries, 1980-2012. JAMA 2014;311:183-192.

9. Li S, Ma C, Xi B. Tobacco control in China: still a long way to go. Lancet 2016;387:1375-1376.

10. O'Donnell MJ, Chin SL, Rangarajan S, Xavier D, Liu L, Zhang H, et al. Global and regional effects of potentially modifiable risk factors associated with acute stroke in 32 countries (INTERSTROKE): a case-control study. Lancet 2016;388:761-775.

11. Hancock DB, Soler Artigas M, Gharib SA, Henry A, Manichaikul $A$, Ramasamy $A$, et al. Genome-wide joint meta-analysis of SNP and SNP-by-smoking interaction identifies novel loci for pulmonary function. PLoS Genet 2012;8:e1003098.

12. Qi $Q$, Chu AY, Kang JH, Jensen MK, Curhan GC, Pasquale LR, et al. Sugar-sweetened beverages and genetic risk of obesity. N Engl J Med 2012;367:1387-1396.

13. Jousilahti P, Rastenyte D, Tuomilehto J, Sarti C, Vartiainen E. 
Parental history of cardiovascular disease and risk of stroke. A prospective follow-up of 14371 middle-aged men and women in Finland. Stroke 1997;28:1361-1366.

14. Tian T, Jin G, Yu C, Lv J, Guo Y, Bian Z, et al. Family history and stroke risk in China: evidence from a large cohort study. J Stroke 2017;19:188-195.

15. Leander K, Hallqvist J, Reuterwall C, Ahlbom A, de Faire U. Family history of coronary heart disease, a strong risk factor for myocardial infarction interacting with other cardiovascular risk factors: results from the Stockholm Heart Epidemiology Program (SHEEP). Epidemiology 2001;12:215-221.

16. Woo D, Khoury J, Haverbusch MM, Sekar P, Flaherty ML, Kleindorfer DO, et al. Smoking and family history and risk of aneurysmal subarachnoid hemorrhage. Neurology 2009;72:69-72.

17. Chen $Z$, Lee $L$, Chen J, Collins R, Wu F, Guo Y, et al. Cohort profile: the Kadoorie Study of Chronic Disease in China (KSCDC). Int J Epidemiol 2005;34:1243-1249.

18. Chen $Z$, Chen J, Collins $R$, Guo $Y$, Peto $R, W u$ F, et al. China Kadoorie Biobank of 0.5 million people: survey methods, baseline characteristics and long-term follow-up. Int J Epidemiol 2011;40:1652-1666.

19. Liu X, Bragg F, Yang L, Kartsonaki C, Guo Y, Du H, et al. Smoking and smoking cessation in relation to risk of diabetes in Chinese men and women: a 9-year prospective study of 0.5 million people. Lancet Public Health 2018;3:e167-e176.

20. Lee CR, North KE, Bray MS, Avery CL, Mosher MJ, Couper DJ, et al. NOS3 polymorphisms, cigarette smoking, and cardiovascular disease risk: the Atherosclerosis Risk in Communities study. Pharmacogenet Genomics 2006;16:891-899.

21. Pezzini A, Grassi M, Del Zotto E, Bazzoli E, Archetti S, Assanelli $D$, et al. Synergistic effect of apolipoprotein $E$ polymorphisms and cigarette smoking on risk of ischemic stroke in young adults. Stroke 2004;35:438-442.

22. Szolnoki Z, Somogyvári F, Kondacs $A$, Szabó $M$, Fodor L, Bene $J$, et al. Evaluation of the modifying effects of unfavourable genotypes on classical clinical risk factors for ischaemic stroke. J Neurol Neurosurg Psychiatry 2003;74:1615-1620.
23. Liu J, Cheng J, Peng J, Han S, Yu L, Nie S. Effects of polymorphisms of heat shock protein 70 gene on ischemic stroke, and interaction with smoking in China. Clin Chim Acta 2007; 384:64-68.

24. Gao X, Yang H, ZhiPing T. Association studies of genetic polymorphism, environmental factors and their interaction in ischemic stroke. Neurosci Lett 2006;398:172-177.

25. Cheng O, Li YK, Lu F, Yin L, Wang YZ, Wei W, et al. Interactions between ACYP2 genetic polymorphisms and environment factors with susceptibility to ischemic stroke in a Han Chinese population. Oncotarget 2017;8:97913-97919.

26. Blann AD, Kirkpatrick U, Devine C, Naser S, McCollum CN. The influence of acute smoking on leucocytes, platelets and the endothelium. Atherosclerosis 1998;141:133-139.

27. Barua RS, Ambrose JA, Saha DC, Eales-Reynolds U. Smoking is associated with altered endothelial-derived fibrinolytic and antithrombotic factors: an in vitro demonstration. Circulation 2002;106:905-908.

28. Nagy J, Demaster EG, Wittmann I, Shultz P, Raij L. Induction of endothelial cell injury by cigarette smoke. Endothelium 1997;5:251-263.

29. Newby DE, Wright RA, Labinjoh $C$, Ludlam CA, Fox KA, Boon $N A$, et al. Endothelial dysfunction, impaired endogenous fibrinolysis, and cigarette smoking: a mechanism for arterial thrombosis and myocardial infarction. Circulation 1999;99: 1411-1415.

30. Wannamethee SG, Lowe GD, Shaper AG, Rumley A, Lennon L, Whincup PH. Associations between cigarette smoking, pipe/ cigar smoking, and smoking cessation, and haemostatic and inflammatory markers for cardiovascular disease. Eur Heart J 2005;26:1765-1773.

31. Renaud S, Blache D, Dumont E, Thevenon C, Wissendanger T. Platelet function after cigarette smoking in relation to nicotine and carbon monoxide. Clin Pharmacol Ther 1984;36:389395.

32. Jha $P$, Peto R. Global effects of smoking, of quitting, and of taxing tobacco. N Engl J Med 2014;370:60-68. 


\section{Supplementary Material}

Members of the China Kadoorie Biobank collaborative group:

International Steering Committee: Junshi Chen, Zhengming Chen (PI), Robert Clarke, Rory Collins, Yu Guo, Liming Li (PI), Jun Lv, Richard Peto, Robin Walters. International Co-ordinating Centre, Oxford: Daniel Avery, Ruth Boxall, Derrick Bennett, Yumei Chang, Yiping Chen, Zhengming Chen, Robert Clarke, Huaidong Du, Simon Gilbert, Alex Hacker, Mike Hill, Michael Holmes, Andri lona, Christiana Kartsonaki, Rene Kerosi, Ling Kong, Om Kurmi, Garry Lancaster, Sarah Lewington, Kuang Lin, John McDonnell, lona Millwood, Qunhua Nie, Jayakrishnan Radhakrishnan, Paul Ryder, Sam Sansome, Dan Schmidt, Paul Sherliker, Rajani Sohoni, Becky Stevens, lain Turnbull, Robin Walters, Jenny Wang, Lin Wang, Neil Wright, Ling Yang, Xiaoming Yang. National Co-ordinating Centre, Beijing: Zheng Bian, Yu Guo, Xiao Han, Can Hou, Jun Lv, Pei Pei, Chao Liu, Yunlong Tan, Canqing Yu. 10 Regional Co-ordinating Centres: Qingdao Centers for Disease Control (CDC): Zengchang Pang, Ruqin Gao, Shanpeng Li, Shaojie Wang, Yongmei Liu, Ranran Du, Yajing Zang, Liang Cheng, Xiaocao Tian, Hua Zhang, Yaoming Zhai, Feng Ning, Xiaohui Sun, Feifei Li. Licang CDC: Silu Lv, Junzheng Wang, Wei Hou. Heilongjiang Provincial CDC: Mingyuan Zeng, Ge Jiang, Xue Zhou. Nangang CDC: Liqiu Yang, Hui He, Bo Yu, Yanjie Li, Qinai Xu,Quan Kang, Ziyan Guo. Hainan Provincial CDC: Dan Wang, Ximin Hu, Jinyan Chen, Yan Fu, Zhenwang Fu, Xiaohuan Wang. Meilan CDC: Min Weng, Zhendong Guo, Shukuan Wu,Yilei Li, Huimei Li, Zhifang Fu. Jiangsu Provincial CDC: Ming Wu, Yonglin Zhou, Jinyi Zhou, Ran Tao, Jie Yang, Jian Su. Suzhou CDC: Fang liu, Jun Zhang, Yihe Hu, Yan Lu, , Liangcai Ma, Aiyu Tang, Shuo Zhang, Jianrong Jin, Jingchao Liu. Guangxi Provincial CDC: Zhenzhu Tang, Naying Chen, Ying Huang. Liuzhou CDC: Mingqiang Li, Jinhuai Meng, Rong Pan, Qilian Jiang, Jian Lan,Yun Liu, Liuping Wei, Liyuan Zhou, Ningyu Chen Ping Wang, Fanwen Meng, Yulu Qin, Sisi Wang. Sichuan Provincial CDC: Xianping Wu, Ningmei Zhang, Xiaofang Chen,Weiwei Zhou. Pengzhou CDC: Guojin Luo, Jianguo Li, Xiaofang Chen, Xunfu Zhong, Jiaqiu Liu, Qiang Sun. Gansu Provincial CDC: Pengfei Ge, Xiaolan Ren, Caixia Dong. Maiji CDC: Hui Zhang, Enke Mao, Xiaoping Wang, Tao Wang, Xi zhang. Henan Provincial CDC: Ding Zhang, Gang Zhou, Shixian Feng, Liang Chang, Lei Fan. Huixian CDC: Yulian Gao, Tianyou He, Huarong Sun, Pan He, Chen Hu, Xukui Zhang, Huifang Wu, Pan He. Zhejiang Provincial CDC: Min Yu, Ruying Hu, Hao Wang. Tongxiang CDC: Yijian Qian, Chunmei Wang, Kaixu Xie, Lingli Chen, Yidan Zhang, Dongxia Pan, Qijun Gu. Hunan Provincial CDC: Yuelong Huang, Biyun Chen, Li Yin, Huilin Liu, Zhongxi Fu, Qiaohua Xu. Liuyang CDC: Xin Xu, Hao Zhang, Huajun Long, Xianzhi Li, Libo Zhang, Zhe Qiu. 
Supplementary Table 1. Multivariable-adjusted HRs (95\% Cls) for incident ischemic stroke according to family history of stroke

\begin{tabular}{|c|c|c|}
\hline \multirow{2}{*}{ Variable } & \multicolumn{2}{|c|}{ Family history of stroke } \\
\hline & No & Yes \\
\hline \multicolumn{3}{|l|}{ Total population } \\
\hline No. of participants & 401,227 & 85,971 \\
\hline No. of ischemic stroke cases & 28,556 & 9,023 \\
\hline Cases per $1,000 \mathrm{PYs}$ & 7.33 & 10.89 \\
\hline \multicolumn{3}{|l|}{$\mathrm{HR}(95 \% \mathrm{Cl})$} \\
\hline Sex adjusted & Ref & $1.25(1.22-1.28)$ \\
\hline Multivariable adjusted ${ }^{*}$ & Ref & $1.19(1.16-1.22)$ \\
\hline \multicolumn{3}{|l|}{ Men } \\
\hline No. of participants & 163,791 & 35,449 \\
\hline No. of ischemic stroke cases & 12,933 & 3,990 \\
\hline Cases per 1,000 PYs & 8.30 & 11.89 \\
\hline \multicolumn{3}{|l|}{$\mathrm{HR}(95 \% \mathrm{Cl})$} \\
\hline Crude & Ref & $1.25(1.21-1.30)$ \\
\hline Multivariable adjusted ${ }^{*}$ & Ref & $1.19(1.15-1.23)$ \\
\hline \multicolumn{3}{|l|}{ Women } \\
\hline No. of participants & 237,436 & 50,522 \\
\hline No. of ischemic stroke cases & 15,623 & 5,033 \\
\hline Cases per $1,000 \mathrm{PYs}$ & 6.69 & 10.21 \\
\hline \multicolumn{3}{|l|}{$\mathrm{HR}(95 \% \mathrm{Cl})$} \\
\hline Crude & Ref & $1.25(1.21-1.29)$ \\
\hline Multivariable adjusted* & Ref & $1.19(1.15-1.23)$ \\
\hline
\end{tabular}

Multivariable model was stratified by age and study area.

$\mathrm{HR}$, hazard ratio; $\mathrm{Cl}$, confidence interval; PY, person-year.

*Adjusted for level of education; marital status; household income; alcohol consumption; smoking status; physical activity; intake frequencies of red meat, fresh fruits, and vegetables; menopause status (only for women); body mass index; and prevalent diabetes and hypertension. 
Supplementary Table 2. Associations between each type of family history and ischemic stroke risk, according to smoking status

\begin{tabular}{llll}
\hline Variable & Never-smokers & Ever-regular smokers & \\
\hline Men & & & \\
Paternal history & $1.17(1.03-1.33)$ & $1.23(1.16-1.31)$ & 0.083 \\
Maternal history & $1.14(1.00-1.31)$ & $1.22(1.14-1.29)$ & 0.122 \\
Parental history & $1.12(1.01-1.25)$ & $1.21(1.15-1.27)$ & 0.021 \\
Sibling history & $1.21(1.03-1.42)$ & $1.22(1.12-1.33)$ & 0.648 \\
Women & & & 0.626 \\
Paternal history & $1.21(1.16-1.27)$ & $1.30(1.06-1.61)$ & 0.233 \\
Maternal history & $1.20(1.15-1.26)$ & $1.37(1.11-1.70)$ & 0.435 \\
Parental history & $1.19(1.15-1.23)$ & $1.29(1.09-1.53)$ & $1.44(1.15-1.80)$ \\
Sibling history & $1.22(1.14-1.30)$ & 0.257 & \\
\hline
\end{tabular}

Values are presented as hazard ratio (95\% confidence interval). Stratified by age and study area and adjusted for level of education; marital status; household income; alcohol consumption; physical activity; intake frequencies of red meat, fresh fruits, and vegetables; menopause status (only for women); body mass index; prevalent hypertension; prevalent diabetes. 